\title{
Touch—High or Low—Is Sometimes More Important Than High Tech
}

\section{Douglas Shinsato, TMT Editor-in-Chief, Technology and Innovation}

"Touch," the ability to connect at a level relevant to those we try to serve, is as important (arguably more important) than deploying the high-level technology our best minds develop. I was treated to an example of this during my visit to Sony Labs several weeks ago to discuss how we might collaborate to accelerate implementation of telehealth technologies.

We agreed that bringing the developed world's healthcare systems into the $21^{\text {st }}$ Century is not an easy task. There are thousands of regulations, millions of healthcare workers whose jobs are at stake, and too many politicians unwilling to tell their voters that their healthcare systems are broken and financially broke.

On the other hand, developing countries are willing to adopt modern telehealth platforms as quickly as possible - but in most cases, they lack the financial resources to move-even at a slower pace.

And the need is great. For example, Bangladesh has a population of 160 million, and nearly half-80 million people living in rural villages-have never seen a medical specialist. The same is true of the Philippines, where nearly half of that country's 100 million citizens have never been to a medical clinic.

During our meeting, Sony Labs' head of renewable energy and microgrid technologies introduced me to his colleague, the head of battery storage. Together, they told me the fascinating story of how Sony combined its divergent expertise to help the fight against Ebola.

Between 2014 and early 2016, nearly 4,000 people in Sierra Leone died out of a confirmed total of 14,000 Ebola infections in that country. In response to a request to 
collect blood samples for Ebola, Sony Labs determined that the following technologies (Figure 1) were required to collect blood samples, which would be sent to the Centers for Disease Control (CDC) in Atlanta, Georgia. To do this, Sony Labs developed a flexible version of solar panels that can be rolled up for easy transport and installation. The energy storage group developed a lithium ferrous ion battery that does not generate heat and can produce up to 2,000 cycles over 10 years. After just 4 hours, there is sufficient energy to set up the rural "healthcare center."

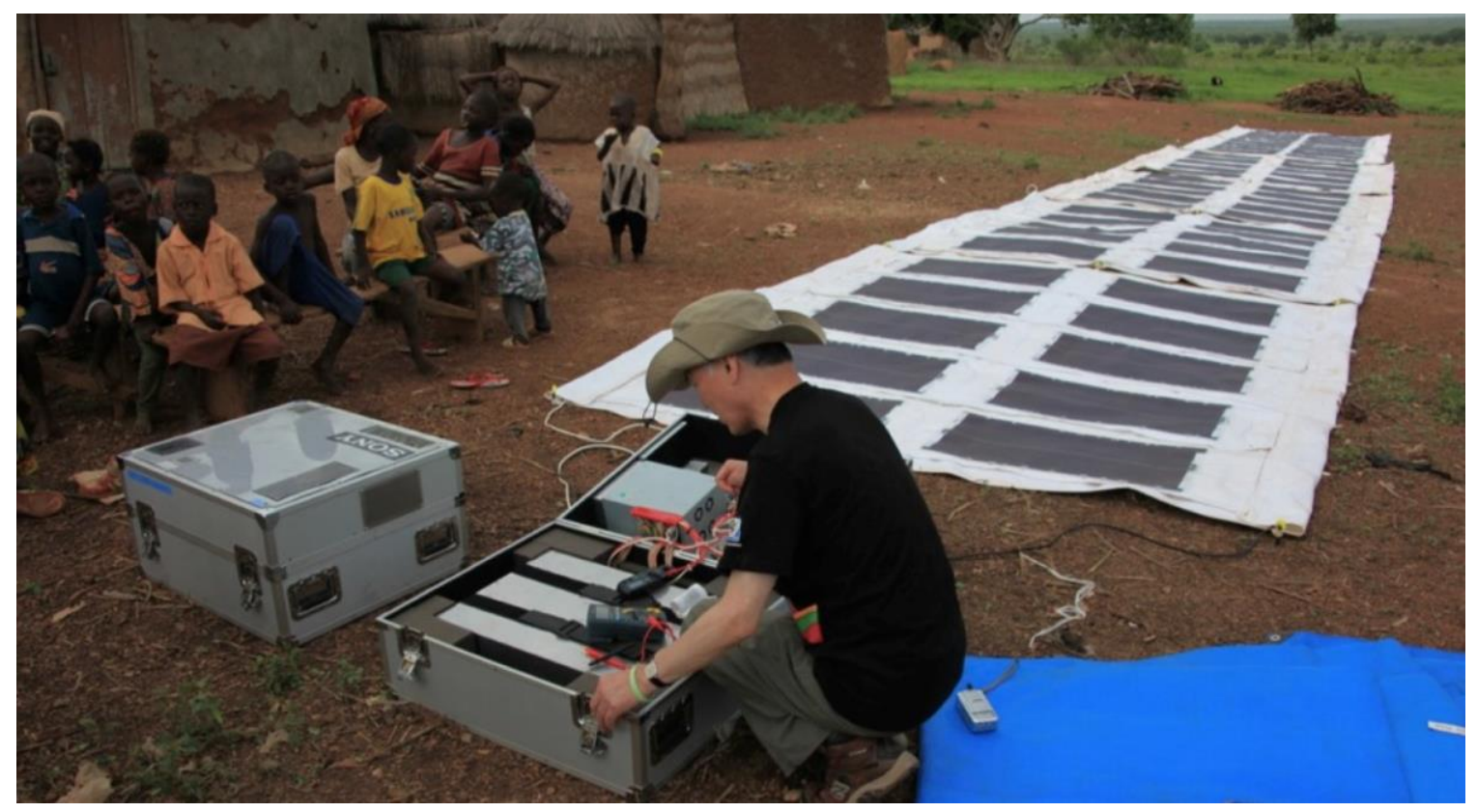

Figure 1. Technologies required to collect blood samples: solar panels to generate electricity in rural areas with no electricity, and batteries to store electricity for night-time use and provide refrigeration for the blood samples.

\section{“Touch"-High or Low}

Sony Labs had set up a low-cost, easy-to-install blood center to collect samples that would aid in the research to prevent such deadly outbreaks in the future.

Notices went out to all the surrounding villages that medical specialists would collect blood samples for Ebola and HIV at no cost. 
No one from the villages showed up.

Then, notices went out that free food and drink would be offered in exchange for a blood sample.

No one showed up.

Someone from the team who understood the concept of High Touch, High Tech came up with a novel idea. What if Sony added a media component to their "healthcare platform" and showed a live soccer (football) match?

Except for those villagers who had visited a town or a city, few people had seen a televised soccer match. Notices went out that everyone was invited and the price of admission was a blood sample. More than a few people showed up, but not enough to collect the number of blood samples required by the Center for Disease Control. And the soccer season was coming to an end.

\section{Enter High (or Low) Touch}

Sony Labs tapped their sister company, Sony Entertainment, which has produced some blockbuster films (e.g., Spider Man, The Da Vinci Code, Casino Royale). Notices went out that the "healthcare center" would be screening never-seen-before action films for the villagers. Again, the price of admission was a blood sample. Figure 2 shows the response. 


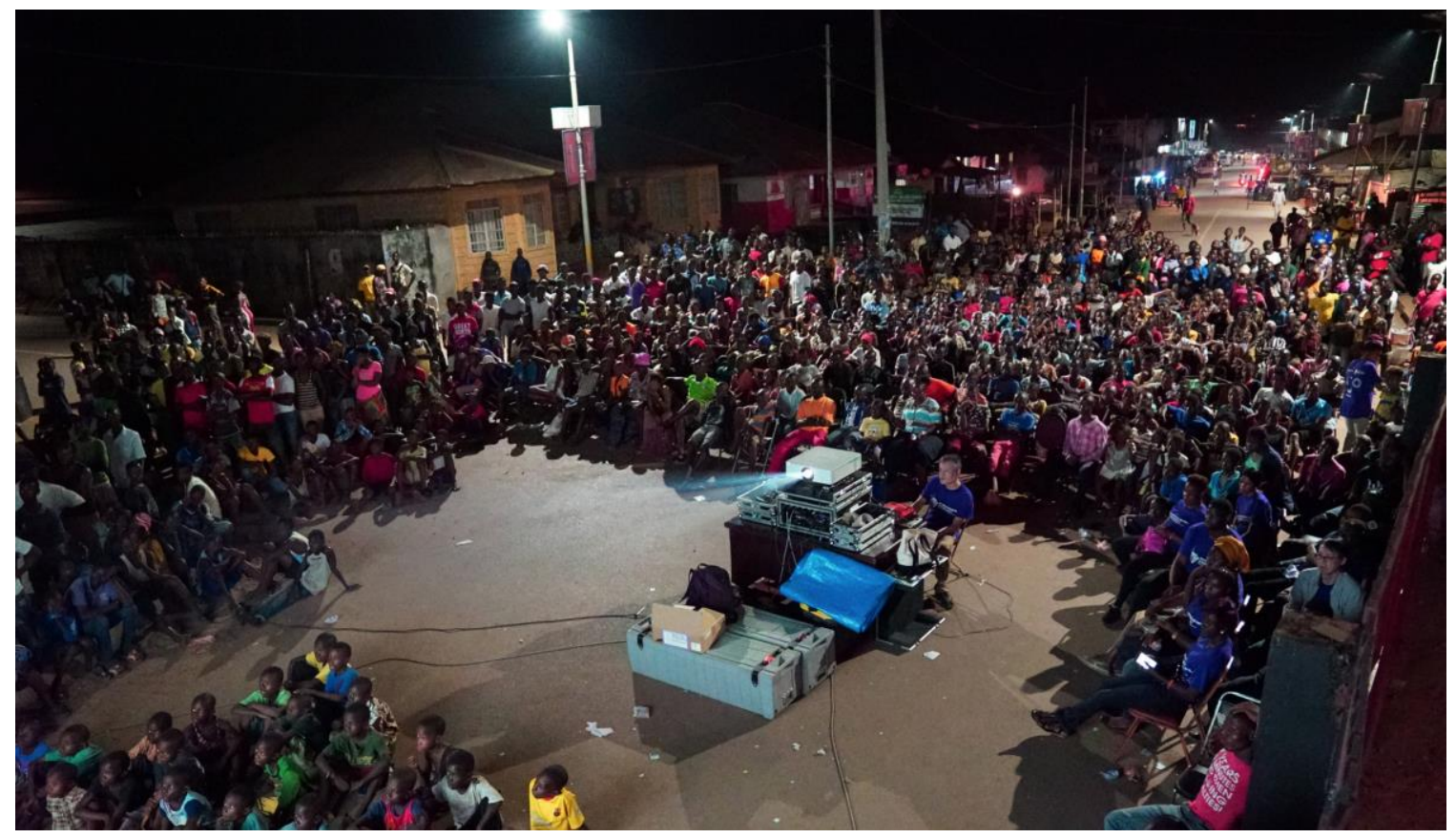

Figure 2. Response to notices that the "healthcare center" would screen never-seen-before action films for the villagers. The price of admission was a blood sample.

\section{Final Note}

Technology provides many benefits, but in many cases, it is simply a powerful enabler. It takes a well-thought-out "Touch" to persuade people to connect with the technology.

The executives at Sony told me that currently the average life expectancy in Sierra Leone is 46 . They agreed that the diffusion of telehealth could increase that to 60 years within a decade.

I asked them, "How would the government ensure that the new gerontocracy would be productive economically and not create a further strain on Sierra Leone's healthcare system?"

Their answer: "We have no idea."

Japan too is facing that problem today with their aging society and fewer tax-paying workers to support increasing overall healthcare costs. 
Technology combined with "Touch" may hold the answer.

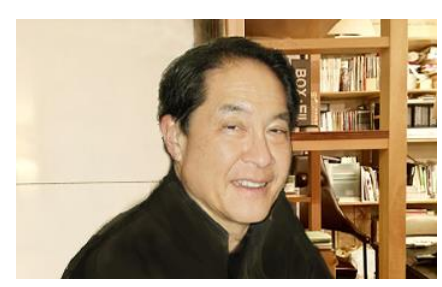

Douglas Shinsato is Co-Editor-in-Chief of Telehealth and Medicine Today and Blockchain in Health Today. He is also Co-Founder of Anthill Ventures, a crossborder incubator and advisor to technology start-ups located in India, Southeast Asia, Europe and the US, focusing on tele-education, telehealth, sustainable food production, and cyber-security.

Category: Clinical Case Study

Tags: Centers for Disease Control, Ebola, high tech, HIV, Sierra Leone, telehealth 